\title{
Erratum to: Structural characterization of the carbohydrate-binding module of NanA sialidase, a pneumococcal virulence factor
}

Lei Yang, Helen Connaris*, Jane A. Potter and Garry L. Taylor

After publication of this article [1], it was noticed there was an error in the Methods section under the subsection: Availability of supporting data.

The text including the error is as follows: "Coordinates and structure factors have been deposited in the Protein Data Bank with accession numbers $4 \mathrm{ZXK}, 4 \mathrm{C} 1 \mathrm{~W}$ and 4CIX for the apo structure, the $3^{1} \mathrm{SL}$ complex and the $6^{1} \mathrm{SL}$ complex, respectively." Instead of 4CIX, it should read $4 \mathrm{C} 1 \mathrm{X}$.

This error has since been updated in the article [1].

Received: 1 October 2015 Accepted: 1 October 2015

Published online: 06 October 2015

\section{References}

1. Yang $L$, Connaris $H$, Potter JA, Taylor GL. Structural characterization of the carbohydrate-binding module of NanA sialidase, a pneumococcal virulence factor. BMC Struct Biol. 2015;15:15. doi:10.1186/s12900-015-0042-4.

* Correspondence: hc6@st-andrews.ac.uk

Biomedical Sciences Research Complex, University of St Andrews, St Andrews, KY16 9ST Fife, UK 